\title{
Periodic solutions for indefinite singular perturbations of the relativistic acceleration
}

\section{Cristian Bereanu}

Faculty of Mathematics, University of Bucharest, 14 Academiei Street, 70190 Bucharest, Romania,

and

Institute of Mathematics 'Simion Stoilow' of the Romanian Academy,

21 Calea Grivitei Street, 010702 Bucharest, Romania

(cristian.bereanu@imar.ro)

\section{Manuel Zamora*}

Departamento de Matemática, Grupo de Investigación en Sistemas Dinámicos y Aplicaciones (GISDA), Universidad del Bío-Bío 5C,

Concepción, Chile (mzamora@ubiobio.cl)

(MS received 16 April 2016; accepted 21 September 2016)

Using the Leray-Schauder degree, we study the existence of solutions for the following periodic differential equation with relativistic acceleration and singular nonlinearity:

$$
\left(\frac{u^{\prime}}{\sqrt{1-u^{\prime 2}}}\right)^{\prime}=\frac{h(t)}{u^{\mu}}, \quad u(0)-u(T)=0=u^{\prime}(0)-u^{\prime}(T),
$$

where $\mu>1$ and the weight $h:[0, T] \rightarrow \mathbb{R}$ is a continuous sign-changing function. There are no a priori estimates on the set of positive solutions (a condition used in general to apply the Leray-Schauder degree), and we prove that no solution of the equation appears on the boundary of an unbounded open set during the deformation to an autonomous problem.

Keywords: singular differential equations; indefinite singularity; periodic solutions; degree theory; continuation theorem

2010 Mathematics subject classification: Primary 34C25

Secondary 34B18

\section{Introduction}

We study the existence of $T$-periodic solutions for the following nonlinear differential equation with relativistic acceleration and singular nonlinearity:

$$
\left(\frac{u^{\prime}}{\sqrt{1-u^{\prime 2}}}\right)^{\prime}=\frac{h(t)}{u^{\mu}}, \quad u(0)-u(T)=0=u^{\prime}(0)-u^{\prime}(T),
$$

* Present address: Departamento de Matemáticas, Universidad de Oviedo, Calle Federico García Lorca 18, 33007 Oviedo, Spain (mzamora@uniovi.es).

(C) 2018 The Royal Society of Edinburgh 
where $\mu>1$ and the weight $h:[0, T] \rightarrow \mathbb{R}$ is a continuous function. A solution of the above problem is a strictly positive function $u \in C^{2}([0, T])$ such that $\max _{[0, T]}\left|u^{\prime}\right|<$ 1 and $u$ verifies the above periodic boundary-value problem.

The main result of this paper is the following theorem.

THEOREM 1.1. Assume that there exists $a \in(0, T)$ such that $h(a)=0, h$ is strictly positive on $[0, a)$ and non-positive on $(a, T]$. If

$$
\int_{0}^{T} h(t) \mathrm{d} t<0 \quad \text { and } \quad \lim _{t \rightarrow a^{-}} \frac{h(t)}{(a-t)^{\mu-1}}=\infty,
$$

the above problem has at least one solution.

First of all, we note that the weight function $h$ must necessarily be sign indefinite, and $\int_{0}^{T} h(t) \mathrm{d} t<0$.

The above periodic boundary-value problem, despite looking simple, is a difficult one. Because the nonlinearity has an indefinite weight and a singularity, there are no a priori estimates on the set of positive solutions, a condition used in general to apply one of the main tools of nonlinear functional analysis: the Leray-Schauder degree. We show only that no solution of the equation appears on the boundary of an unbounded open set during the deformation.

To overcome this problem, we introduce a new strategy, together with a homotopy with an autonomous equation. We prove a continuation theorem (theorem 2.3) for problems of the type

$$
\left(\frac{u^{\prime}}{\sqrt{1-u^{\prime 2}}}\right)^{\prime}=h(t) g(u), \quad u(0)-u(T)=0=u^{\prime}(0)-u^{\prime}(T),
$$

where the weight $h$ is such that

$$
\bar{h}=T^{-1} \int_{0}^{T} h(t) \mathrm{d} t \neq 0,
$$

and $g: \mathbb{R} \rightarrow \mathbb{R}$ is continuous. The main idea is to consider for $\lambda \in[0,1]$ the homotopy

$$
\begin{gathered}
\left(\frac{u^{\prime}}{\sqrt{1-u^{\prime 2}}}\right)^{\prime}=(\lambda h(t)-(1-\lambda) \bar{h}) g(u)+(1-\lambda) \bar{h}, \\
u(0)-u(T)=0=u^{\prime}(0)-u^{\prime}(T) .
\end{gathered}
$$

If there exists $0<\varepsilon<R$ such that $g(\varepsilon)>1>g(R)$, and $J \subset[0, T]$ closed such that the above problem has no solution on $\partial V$ for all $\lambda \in[0,1]$, where

$$
V=\{u:[0, T] \rightarrow \mathbb{R}: u \text { continuous with } u(0)=u(T), \varepsilon<u<R \text { on } J\},
$$

then the above problem for $\lambda=1$ has at least one solution in $V$. The main tool in the proof is a continuation theorem from [14] (see also $[2,11]$ ) in which a homotopy is made to an arbitrary autonomous equation and one takes advantage of the $S^{1}$ invariance of the corresponding periodic problem to compute the associated LeraySchauder degree.

There is a large literature concerning nonlinear super- and sublinear problems with a weight function having an indefinite sign (see, for example, $[1,6,7,12]$ ). 
However, fewer results concerning problems with singularity and indefinite weight seem to be available. Our paper is motivated by the papers [8,15]. In both these papers, pure ordinary differential equation strategies are used in the proofs. In the first one the weight satisfies strong symmetry conditions, and in the second the weight has only non-degenerate zeros or is piecewise constant. The existence of periodic solutions for singular nonlinearities with indefinite weight and Newtonian acceleration is considered for the first time in [9].

Our paper is structured as follows. In $\S 2$ we prove our continuation theorem. In $\S 3$ we apply the continuation theorem to a modified problem, and in $\S 4$ we prove that the solution of a particular modified problem is also solution of the main periodic problem. For results concerning periodic solutions of nonlinear perturbation of the relativistic acceleration, see, for example, $[4,5,10]$.

\section{Homotopy and degree}

To construct the fixed-point operator we need some notation. Let $C$ denote the Banach space of continuous functions on $[0, T]$ endowed with the uniform norm $\|\cdot\|_{\infty}$. We consider the closed subspace

$$
C_{T}=\{u \in C: u(0)=u(T)\} .
$$

The open ball of centre 0 and radius $r$ is denoted by $B_{r}$. We denote by $P, Q: C \rightarrow C$ the continuous projectors

$$
P u(t)=u(0), \quad Q u(t)=T^{-1} \int_{0}^{T} u(s) \mathrm{d} s=\bar{u} \quad(t \in[0, T]) .
$$

On the other hand, let $H: C \rightarrow C$ be the continuous linear operator given by

$$
H u(t)=\int_{0}^{t} u(s) \mathrm{d} s \quad(t \in[0, T]) .
$$

Throughout the paper we use the following notation:

$$
\phi(s)=\frac{s}{\sqrt{1-s^{2}}} \quad(s \in(-1,1)) .
$$

Let $Q_{\phi}: C \rightarrow \mathbb{R}$ be the continuous function determined by the relation

$$
Q \circ \phi^{-1} \circ\left(I-Q_{\phi}\right) \circ u=0 \text { for all } u \in C .
$$

We need the following fixed-point lemma (see [3, proposition 2]).

LEMma 2.1. Assume that $F: C \rightarrow C$ is continuous and takes bounded sets into bounded sets. Then, $u$ is a solution of the abstract periodic problem

$$
\left(\phi\left(u^{\prime}\right)\right)^{\prime}=F(u), \quad u(0)-u(T)=0=u^{\prime}(0)-u^{\prime}(T),
$$

if and only if $u \in C_{T}$ is a fixed point of the completely continuous operator $M: C_{T} \rightarrow$ $C_{T}$ given by

$$
M=P+Q F+H \circ \phi^{-1} \circ\left(I-Q_{\phi}\right) \circ[H(I-Q) F] .
$$


Using exactly the same proof as in [14, theorem 4.1], one has the following continuation result.

Lemma 2.2. Consider the periodic problems

$$
\left(\phi\left(u^{\prime}\right)\right)^{\prime}=f(t, u, \lambda), \quad u(0)-u(T)=0=u^{\prime}(0)-u^{\prime}(T),
$$

where $f:[0, T] \times \mathbb{R} \times[0,1]$ is continuous and

$$
f(t, u, 0)=f_{0}(u)
$$

is independent of $t$. Let $\Omega$ be an open bounded subset in $C_{T}$ such that for each $\lambda \in[0,1]$ the above problem has no solution on $\partial \Omega$. Then,

$$
d_{\mathrm{LS}}\left[I-M_{1}, \Omega, 0\right]=-d_{\mathrm{B}}\left[f_{0}, \Omega \cap \mathbb{R}, 0\right],
$$

where $M_{1}$ is the fixed-point operator of the above problem for $\lambda=1$.

Next, for each $\lambda \in[0,1]$, we consider the periodic problem

$$
\left.\begin{array}{c}
\left(\phi\left(u^{\prime}\right)\right)^{\prime}=(\lambda h(t)-(1-\lambda) \bar{h}) g(u)+(1-\lambda) \bar{h}, \\
u(0)-u(T)=0=u^{\prime}(0)-u^{\prime}(T),
\end{array}\right\}
$$

where $h \in C$ and $g: \mathbb{R} \rightarrow \mathbb{R}$ is continuous. The main result of this section is the following theorem.

Theorem 2.3. Assume that $\bar{h} \neq 0$ and there exists $0<\varepsilon<R$ such that $g(\varepsilon)>$ $1>g(R)$. Let $J \subset[0, T]$ be closed and

$$
V=\left\{u \in C_{T}: \varepsilon<u<R \text { on } J\right\} .
$$

If, for each $\lambda \in[0,1]$, (2.1) has no solution on $\partial V$, then, for $\lambda=1$, (2.1) has at least one solution in $V$.

Proof. Let $u \in \bar{V}$ be a solution of (2.1). Then, by the fundamental theorem of calculus, we have

$$
|u(t)| \leqslant R+\int_{0}^{T}\left|u^{\prime}(s)\right| \mathrm{d} s<R+T
$$

for each $t \in[0, T]$. Taking the open bounded set $\Omega=V \cap B_{R+T}$, it follows that (2.1) has no solution on $\partial \Omega$, Hence, the homotopy invariance of Leray-Schauder degree, together with the above lemma, implies that

$$
d_{\mathrm{LS}}\left[I-M_{1}, \Omega, 0\right]=d_{\mathrm{LS}}\left[I-M_{0}, \Omega, 0\right]=-d_{\mathrm{B}}[\bar{h}(1-g),(\varepsilon, R), 0],
$$

where $M_{\lambda}$ is the fixed-point operator corresponding to (2.1). It follows that $d_{\mathrm{LS}}[I-$ $\left.M_{1}, \Omega, 0\right] \neq 0$, and the existence property of the Leray-Schauder degree concludes the proof. 


\section{A modified problem}

In this section we assume that

$$
\bar{h}<0 .
$$

For each $0<\delta<1$ we define a truncation function $g_{\delta}: \mathbb{R} \rightarrow \mathbb{R}$ such that $g_{\delta}$ is smooth on $\mathbb{R}$ and is non-increasing and bounded by $1+\delta^{-\mu}$, and $g_{\delta}(u)=u^{-\mu}$ for any $u \geqslant \delta$. Consider the following family of periodic problems:

$$
\left.\begin{array}{c}
\left(\phi\left(u^{\prime}\right)\right)^{\prime}=(\lambda h(t)-(1-\lambda) \bar{h}) g_{\delta}(u)+(1-\lambda) \bar{h}, \\
u(0)-u(T)=0=u^{\prime}(0)-u^{\prime}(T) .
\end{array}\right\}
$$

LEMmA 3.1. There exists $R>1+T$ such that, for any solution $u$ of (3.1), one has

$$
\max _{[0, T]} u<R .
$$

Proof. Let

$$
H_{+}=\int_{0}^{a} h(t) \mathrm{d} t, \quad H_{-}=-\int_{a}^{T} h(t) \mathrm{d} t .
$$

We take $R>1+T$ sufficiently large such that

$$
H_{+}-\left(\frac{x-T}{x}\right)^{\mu} H_{-}+T \bar{h}\left(1-(x-T)^{\mu}\right)>0, \quad H_{+}-\left(\frac{x-T}{x}\right)^{\mu} H_{-}<0,
$$

for all $x \geqslant R$. Assume that $u$ is a solution of $(3.1)$ such that $M_{u}=\max _{[0, T]} u \geqslant$ $R$. By integrating both sides of (3.1) over $[0, T]$ and taking into account that $\max _{[0, T]} u-\min _{[0, T]} u<T$, we obtain that

$$
0 \leqslant \lambda H_{+}-\lambda\left(\frac{M_{u}-T}{M_{u}}\right)^{\mu} H_{-}-(1-\lambda) T \bar{h}+(1-\lambda) \bar{h} T\left(M_{u}-T\right)^{\mu} .
$$

It follows that

$$
0 \leqslant H_{+}-\left(\frac{M_{u}-T}{M_{u}}\right)^{\mu} H_{-},
$$

which is a contradiction of the choice of $R$.

Lemma 3.2. Assume that there exists $R>0$ such that $u \in B_{R}$ for each solution $u$ of (3.1). There exists $K>0$ such that, for any solution $u$ of (3.1), one has

$$
\left\|\frac{\phi\left(u^{\prime}\right)}{g_{\delta}(u)}\right\|_{\infty} \leqslant K
$$

Proof. Let $u \in B_{R}$ be a solution of (3.1). Let $t_{m} \in[0, T]$ be the point where $u$ attains the maximum value on $[0, T]$. From the periodic boundary conditions one has that $u^{\prime}\left(t_{m}\right)=0$. Multiplying both sides of (3.1) by $g_{\delta}(u)^{-1}$ and integrating by parts, we deduce that

$$
\frac{\phi\left(u^{\prime}\right)}{g_{\delta}(u)}=\int_{t_{m}}^{t} \phi\left(u^{\prime}\right)\left[\frac{1}{g_{\delta}(u(s))}\right]^{\prime} \mathrm{d} s+\int_{t_{m}}^{t}(\lambda h(s)-(1-\lambda) \bar{h}) \mathrm{d} s+(1-\lambda) \bar{h} \int_{t_{m}}^{t} \frac{\mathrm{d} s}{g_{\delta}(u)}
$$


for all $t \in[0, T]$. Taking into account that $g_{\delta}$ is a non-increasing function, one can easily to check that

$$
\phi\left(u^{\prime}(s)\right) \frac{\mathrm{d}}{\mathrm{d} t}\left[\frac{1}{g_{\delta}(u(t))}\right]_{t=s} \geqslant 0
$$

for all $s \in[0, T]$. It follows that

$$
\frac{\phi\left(u^{\prime}\right)}{g_{\delta}(u)} \leqslant K \quad \text { on }\left[0, t_{m}\right], \quad \frac{\phi\left(u^{\prime}\right)}{g_{\delta}(u)} \geqslant-K \quad \text { on }\left[t_{m}, T\right],
$$

where $K:=\|h\|_{1}+T|\bar{h}|\left(1+R^{\mu}\right)$. The conclusion now follows from the periodic boundary conditions.

Lemma 3.3. There exists $0<\varepsilon<1$ such that if $0<\delta \leqslant \varepsilon$ and $u$ is a solution of (3.1) then $\min _{[0, a / 2]} u \neq \varepsilon$.

Proof. Let $M_{0}>0$ be such that

$$
\begin{gathered}
\int_{a / 2}^{3 a / 4} \phi^{-1}\left(\frac{\left(h_{0}+\bar{h} M^{\mu}\right)\left(s-\frac{1}{2} a\right)}{M^{\mu}}\right) \mathrm{d} s>M, \\
\int_{0}^{a / 2} \phi^{-1}\left(\frac{\left(h_{0}+\bar{h} M^{\mu}\right)\left(\frac{1}{2} a-s\right)}{M^{\mu}}\right) \mathrm{d} s>M,
\end{gathered}
$$

for all $M<M_{0}$, where $h_{0}:=\min \left\{\min _{[0,3 a / 4]} h,|\bar{h}|\right\}>0$. Moreover, we can assume that

$$
\frac{h_{0}}{M_{0}^{\mu}}+\bar{h}>0
$$

We take $R>T+1$ as in lemma 3.1 and $K>0$ verifying lemma 3.2, and we consider $0<\varepsilon<\min \left\{M_{0}, 1\right\}$ such that

$$
K<h_{0} M_{0}^{\mu} \int_{\varepsilon}^{M_{0}} \frac{\mathrm{d} s}{s^{\mu}}-|\bar{h}|(T+1)^{\mu+1} .
$$

Let $\delta \in(0, \varepsilon]$ be fixed. Assume that there exists a solution $u$ of (3.1) such that $\min _{[0, a / 2]} u=\varepsilon$. Let $t_{*} \in\left[0, \frac{1}{2} a\right]$ be such that $u\left(t_{*}\right)=\varepsilon$. Using lemma 3.1, one has that $\max _{[0, T]} u<R$, and then

$$
\min _{[0, T]} u>\max _{[0, T]} u-T>\varepsilon-T>-R .
$$

Hence,

$$
\|u\|_{\infty}<R
$$

We distinguish two cases.

CASE $1\left(t_{*} \in\left[0, \frac{1}{2} a\right)\right)$. Observe that $u^{\prime}\left(t_{*}\right) \geqslant 0$. We claim that

$$
\max _{\left[t_{*}, 3 a / 4\right]} u \geqslant M_{0}
$$

Indeed, if we assume that $u<M_{0}$ on $\left[t_{*}, \frac{3}{4} a\right]$, then one has that $\left(\phi\left(u^{\prime}\right)\right)^{\prime}>0$ on $\left[t_{*}, \frac{3}{4} a\right]$. Since $u^{\prime}\left(t_{*}\right) \geqslant 0$, we have $u^{\prime}(t) \geqslant 0$ and $\varepsilon \leqslant u(t)$ for $t \in\left[t_{*}, \frac{3}{4} a\right]$ and 
$u\left(\frac{3}{4} a\right)=\max _{\left[t_{*}, 3 a / 4\right]} u$. Multiplying both sides of (3.1) by $u^{\mu}$ and integrating from $t_{*}$ to $t \in\left[t_{*}, \frac{3}{4} a\right]$, we deduce that

$$
\phi\left(u^{\prime}(t)\right) \geqslant \frac{\left(h_{0}+\bar{h} u\left(\frac{3}{4} a\right)^{\mu}\right)\left(t-t_{*}\right)}{u^{\mu}(t)} \text { for all } t \in\left[t_{*}, \frac{3}{4} a\right] .
$$

Since $\phi$ is an increasing homeomorphism, it follows that

$$
u^{\prime}(t) \geqslant \phi^{-1}\left(\frac{\left(h_{0}+\bar{h} u\left(\frac{3}{4} a\right)^{\mu}\right)\left(t-t_{*}\right)}{u\left(\frac{3}{4} a\right)^{\mu}}\right) \quad \text { for all } t \in\left[t_{*}, \frac{3}{4} a\right] .
$$

Integrating the latter inequality over $\left[t_{*}, \frac{3}{4} a\right]$, we obtain that

$$
\begin{aligned}
u\left(\frac{3 a}{4}\right) & \geqslant \int_{t_{*}}^{3 a / 4} \phi^{-1}\left(\frac{\left(h_{0}+\bar{h} u\left(\frac{3}{4} a\right)^{\mu}\right)\left(t-t_{*}\right)}{u\left(\frac{3}{4} a\right)^{\mu}}\right) \mathrm{d} t \\
& \geqslant \int_{a / 2}^{3 a / 4} \phi^{-1}\left(\frac{\left(h_{0}+\bar{h} u\left(\frac{3}{4} a\right)^{\mu}\right)\left(t-\frac{1}{2} a\right)}{u\left(\frac{3}{4} a\right)^{\mu}}\right) \mathrm{d} t,
\end{aligned}
$$

a contradiction of the choice of $M_{0}$.

Since $\max _{\left[t_{*}, 3 a / 4\right]} u \geqslant M_{0}$, we can define $b_{*} \in\left(t_{*}, \frac{3}{4} a\right]$ such that $u\left(b_{*}\right)=M_{0}$ and $\varepsilon \leqslant u \leqslant M_{0}$ on $\left[t_{*}, b_{*}\right]$. Then $\left(\phi\left(u^{\prime}\right)\right)^{\prime}>0$ and $u^{\prime} \geqslant 0\left(\right.$ since $\left.u^{\prime}\left(t_{*}\right) \geqslant 0\right)$ on $\left[t_{*}, b_{*}\right]$. Moreover,

$$
\left(\phi\left(u^{\prime}\right)\right)^{\prime} u^{\prime} \geqslant \frac{h_{0} u^{\prime}}{u^{\mu}}+(1-\lambda) \bar{h} u^{\prime} \quad \text { on }\left[t_{*}, b_{*}\right] .
$$

Integrating from $t_{*}$ to $t \in\left[t_{*}, b_{*}\right]$, we obtain

$$
\phi\left(u^{\prime}(t)\right) \geqslant h_{0} \int_{\varepsilon}^{u(t)} \frac{\mathrm{d} s}{s^{\mu}}+\bar{h}(T+1) \quad \text { for all } t \in\left[t_{*}, b_{*}\right] .
$$

Since $\|u\|_{\infty}<R$ and $u^{\mu}=g_{\delta}(u)^{-1}$ on $\left[t_{*}, b_{*}\right]$, multiplying both sides of the above inequality by $u^{\mu}$ and applying lemma 3.2 yields

$$
K \geqslant h_{0} u^{\mu}(t) \int_{\varepsilon}^{u(t)} \frac{\mathrm{d} s}{s^{\mu}}-|\bar{h}|(T+1)^{\mu+1} \quad \text { for all } t \in\left[t_{*}, b_{*}\right],
$$

which contradicts the choice of $K$ taking $t=b_{*}$.

CASE $2\left(t_{*}=\frac{1}{2} a\right)$. Observe that $u^{\prime}\left(t_{*}\right) \leqslant 0$. We claim that

$$
\max _{\left[0, t_{*}\right]} u \geqslant M_{0}
$$

Assume that $u<M_{0}$ on $\left[0, t_{*}\right]$. This implies that $\left(\phi\left(u^{\prime}\right)\right)^{\prime}>0$ on $\left[0, t_{*}\right]$. Since $u^{\prime}\left(t_{*}\right) \leqslant 0$, we have $u^{\prime}(t) \leqslant 0$ and $\varepsilon \leqslant u(t)$ for all $t \in\left[0, t_{*}\right]$. Arguing as in case 1 , multiplying both sides of $(3.1)$ by $u^{\mu}$, integrating from $t \in\left[0, t_{*}\right]$ to $t_{*}$ and using that $u(0)=\max _{\left[0, t_{*}\right]} u$, we obtain

$$
-u^{\prime}(t) \geqslant \phi^{-1}\left(\frac{\left(h_{0}+u(0)^{\mu} \bar{h}\right)\left(t_{*}-t\right)}{u(0)^{\mu}}\right) \text { for all } t \in\left[0, t_{*}\right] .
$$

Integrating the latter inequality over $\left[0, t_{*}\right]$, we obtain a contradiction of the choice of $M_{0}$. 
Finally, since $\max _{\left[0, t_{*}\right]} u \geqslant M_{0}$, we can define $a_{*} \in\left[0, t_{*}\right)$ such that $u\left(a_{*}\right)=M_{0}$ and $\varepsilon \leqslant u(t) \leqslant M_{0}$ for all $t \in\left[a_{*}, t_{*}\right]$. Then $\left(\phi\left(u^{\prime}\right)\right)^{\prime}>0$ and $u^{\prime}(t) \leqslant 0$ (since $\left.u^{\prime}\left(t_{*}\right) \leqslant 0\right)$ on $\left[a_{*}, t_{*}\right]$. Moreover,

$$
\left(\phi\left(u^{\prime}\right)\right)^{\prime} u^{\prime} \leqslant \frac{h_{0} u^{\prime}}{u^{\mu}}+(1-\lambda) \bar{h} u^{\prime} \quad \text { on }\left[a_{*}, t_{*}\right] .
$$

By integrating from $t \in\left[a_{*}, t_{*}\right]$ to $t_{*}$ we have

$$
\phi\left(u^{\prime}(t)\right) \leqslant-h_{0} \int_{\varepsilon}^{u(t)} \frac{\mathrm{d} s}{s^{\mu}}+|\bar{h}|(T+1) \quad \text { for all } t \in\left[a_{*}, t_{*}\right] .
$$

Since $\|u\|_{\infty}<R$ and $u^{\mu}=g_{\delta}(u)^{-1}$ on $\left[a_{*}, t_{*}\right]$, multiplying both sides of the above inequality by $u^{\mu}$ yields

$$
-K \leqslant-h_{0} u^{\mu}(t) \int_{\varepsilon}^{u(t)} \frac{\mathrm{d} s}{s^{\mu}}+|\bar{h}|(T+1)^{1+\mu} \quad \text { for all } t \in\left[a_{*}, t_{*}\right],
$$

which contradicts the choice of $K$ taking $t=a_{*}$.

The main result of this section is the following existence result concerning the modified problem.

Proposition 3.4. There exists $0<\varepsilon<1$ such that if $0<\delta \leqslant \varepsilon$, then the problem

$$
\left(\phi\left(u^{\prime}\right)\right)^{\prime}=h(t) g_{\delta}(u), \quad u(0)-u(T)=0=u^{\prime}(0)-u^{\prime}(T),
$$

has at least one solution $u$ with $\min _{[0, a / 2]} u>\varepsilon$.

Proof. This follows immediately from lemmas 3.1 and 3.3 and theorem 2.3.

\section{Proof of the main result}

We take $R>T+1$ as in lemma 3.1, $K>0$ verifying lemma 3.2 and $\varepsilon>0$ given by proposition 3.4. Using the assumptions upon $h$, there exists $a_{*} \in\left(\frac{1}{2} a, a\right)$ such that

$$
h_{0} \varepsilon^{\mu} \int_{2 \delta}^{\varepsilon} \frac{\mathrm{d} s}{s^{\mu}}>K,
$$

where $h_{0}=\min _{\left[0, a_{*}\right]} h$, and $\delta=a-a_{*}$ is such that $2 \delta<\varepsilon$. Consider $u$ given by proposition 3.4 with $\delta$ defined above. We will show that $u \geqslant \delta$ on $[0, T]$. This means that $u$ is a solution to our main periodic problem.

Assume by contradiction that $\min _{[0, T]} u<\delta$. Using that $\min _{[0, a / 2]} u>\varepsilon$ and the periodic boundary conditions, it follows that there exists $t_{*} \in\left(\frac{1}{2} a, T\right)$ such that $\min _{[0, T]} u=u\left(t_{*}\right)$.

We have three cases.

CASE $1\left(t_{*} \in\left(\frac{1}{2} a, a_{*}\right]\right)$. Since $u\left(\frac{1}{2} a\right)>\varepsilon$, there exists $\tilde{t} \in\left(\frac{1}{2} a, t_{*}\right)$ such that $u(\tilde{t})=$ $\varepsilon$. Moreover, using the modified problem, one has that $\left(\phi\left(u^{\prime}\right)\right)^{\prime} \geqslant 0$ on $\left[\frac{1}{2} a, t_{*}\right]$, which implies that $u^{\prime}$ is non-increasing on $\left[\frac{1}{2} a, t_{*}\right]$. Then, since $u^{\prime}\left(t_{*}\right)=0$, it follows that $u^{\prime} \leqslant 0$ on $\left[\frac{1}{2} a, t_{*}\right]$ and

$$
\left(\phi\left(u^{\prime}\right)\right)^{\prime} u^{\prime} \leqslant h_{0} g_{\delta}(u) u^{\prime} \quad \text { on }\left[\frac{1}{2} a, t_{*}\right]
$$


Integrating, we deduce that

$$
\phi\left(u^{\prime}(t)\right) \leqslant-h_{0} \int_{u\left(t_{*}\right)}^{u(t)} g_{\delta}(s) \mathrm{d} s \quad \text { for all } t \in\left[\frac{1}{2} a, t_{*}\right] .
$$

Multiplying by $g_{\delta}(u)^{-1}$ in the latter inequality and applying lemma 3.2 (note that $\left.\|u\|_{\infty}<R\right)$ we deduce that

$$
-K \leqslant \frac{-h_{0}}{g_{\delta}(u(t))} \int_{\delta}^{u(t)} g_{\delta}(s) \mathrm{d} s \quad \text { for all } t \in\left[\frac{1}{2} a, t_{*}\right]
$$

which contradicts the choice of $\delta$ taking $t=\tilde{t}$.

CASE $2\left(t_{*} \in\left(a_{*}, a\right]\right)$. In this case we use a strategy inspired by [13]. Our first task will consist in observing that

$$
u\left(a_{*}\right) \geqslant 2 \delta .
$$

We use a contradiction argument and assume that $u\left(a_{*}\right)<2 \delta$. Since $\left(\phi\left(u^{\prime}\right)\right)^{\prime} \geqslant 0$ on $[0, a]$ and $u^{\prime}\left(t_{*}\right)=0$, we have $u^{\prime}(t) \leqslant 0$ for all $t \in\left[0, t_{*}\right]$. Using the modified problem, multiplying by $u^{\prime}$ and integrating from $t \in\left[0, a_{*}\right]$ to $a_{*}$, we obtain

$$
\phi\left(u^{\prime}(t)\right) \leqslant-h_{0} \int_{u\left(a_{*}\right)}^{u(t)} g_{\delta}(s) \mathrm{d} s \text { for all } t \in\left[0, a_{*}\right] .
$$

On the other hand, since $u\left(\frac{1}{2} a\right)>\varepsilon$, there exists $\tilde{t} \in\left(\frac{1}{2} a, a_{*}\right)$ such that $u(\tilde{t})=$ $\varepsilon$. Multiplying by $g_{\delta}(u)^{-1}$ in both sides of the inequality above and applying lemma 3.2 , we deduce that

$$
-K \leqslant \frac{-h_{0}}{g_{\delta}(u(t))} \int_{2 \delta}^{u(t)} g_{\delta}(s) \mathrm{d} s \text { for all } t \in\left[0, a_{*}\right] .
$$

This is a contradiction of the choice of $a_{*}$ taking $t=\tilde{t}$.

Next, let $t_{0} \in\left[a_{*}, t_{*}\right)$ be such that $u\left(t_{0}\right)=2 \delta$. A direct integration in the modified problem shows that

$$
-\phi\left(u^{\prime}(t)\right)=\int_{t}^{t_{*}} h(s) g_{\delta}(u) \mathrm{d} s \leqslant\left(1+\delta^{-\mu}\right) \int_{t}^{t_{*}} h(s) \mathrm{d} s
$$

for all $t \in\left[0, t_{*}\right]$. Thus,

$$
-u^{\prime}(t) \leqslant \phi^{-1}\left(\left(1+\delta^{-\mu}\right) \int_{t_{*}}^{t} h(s) \mathrm{d} s\right) \quad \text { for all } t \in\left[t_{0}, t_{*}\right] .
$$

We integrate over $\left[t_{0}, t_{*}\right]$ obtaining that

$$
2 \delta-u\left(t_{*}\right)<t_{*}-t_{0} \leqslant a-a_{*}=\delta .
$$

However, this contradicts that $u\left(t_{*}\right)<\delta$.

CASE $3\left(t_{*} \in(a, T]\right)$. From the modified problem it follows that $\left(\phi\left(u^{\prime}\right)\right)^{\prime} \leqslant 0$ on $[a, T]$, which implies that $u$ is constant on $[a, T]$. Hence,

$$
\delta>u\left(t_{*}\right)=u(T)=u(0)>\varepsilon,
$$

which is a contradiction. The proof is complete. 


\section{Acknowledgements}

We thank the referee for comments. C.B. is partly supported by CNCS-UEFISCDI Project no. PN-II-RU-TE-2014-4-0007. M.Z. is supported by FONDECYT Project no. 11140203.

\section{References}

1 C. Bandle, M. A. Pozio and A.Tesei. Existence and uniqueness of solutions of nonlinear problems. Math. Z. 199 (1988), 257-278.

2 T. Bartsch and J. Mawhin. The Leray-Schauder degree of $S^{1}$-equivariant operators associated to autonomous neutral equations in spaces of periodic functions. J. Diff. Eqns 92 (1991), 90-99.

3 C. Bereanu and J. Mawhin. Existence and multiplicity results for some nonlinear problems with singular $\phi$-Laplacian. J. Diff. Eqns 243 (2007), 536-557.

4 C. Bereanu, P. Jebelean and J. Mawhin. Multiple solutions for Neumann and periodic problems with singular $\phi$-Laplacian. J. Funct. Analysis 261 (2011), 3226-3246.

5 C. Bereanu, D. Gheorghe and M. Zamora. Periodic solutions for the singular perturbations of the singular $\phi$-Laplacian operator. Commun. Contemp. Math. 15 (2013), 1250063.

6 H. Berestycki, I. Capuzzo-Dolcetta and L. Nirenberg. Variational methods for indefinite superlinear homogeneous elliptic problems. NoDEA. Nonlin. Diff. Eqns Applic. 2 (1995), $553-572$.

$7 \quad$ A. Boscaggin and F. Zanolin. Pairs of positive periodic solutions of second order nonlinear equations with indefinite weight. J. Diff. Eqns 252 (2012), 2900-2921.

8 A. Boscaggin and F. Zanolin. Second-order ordinary differential equations with indefinite weight: the Neumann boundary value problem. Annali Mat. Pura Appl. 194 (2015), 451478.

9 J. L. Bravo and P. J. Torres. Periodic solutions of a singular equation with indefinite weight. Adv. Nonlin. Studies 10 (2010), 927-938.

$10 \mathrm{H}$. Brézis and J. Mawhin. Periodic solutions of the forced relativistic pendulum. Diff. Integ. Eqns 23 (2010), 801-810.

11 A. Capietto, J. Mawhin and F. Zanolin. Continuation theorems for periodic perturbations of autonomous systems. Trans. Am. Math. Soc. 329 (1992), 41-72.

12 D. G. De Figueiredo, J.-P. Gossez and P. Ubilla. Local superlinearity and sublinearity for indefinite semilinear elliptic problems. J. Funct. Analysis 199 (2003), 452-467.

13 A. C. Lazer and S. Solimini. On periodic solutions of nonlinear differential equations with singularities. Proc. Am. Math. Soc. 99 (1987), 109-114.

14 R. Manasevich and J. Mawhin. Periodic solutions for nonlinear systems with $p$-Laplacianlike operators. J. Diff. Eqns 145 (1998), 367-393.

15 A. J. Ureña. Periodic solutions of singular equations with indefinite weight. Topolog. Meth. Nonlin. Analysis 47 (2016), 55-72. 